\title{
Relevancia del E-commerce para el sector confecciones de la ciudad de Pereira
}

\author{
Juliana M. Arias Molina' ; Valeria Parra Flórez²
}

RESUMEN El presente documento establece una propuesta para tratar de determinar cuál es la importancia que tiene el E-commerce para el mejoramiento de la comercialización de productos en el sector confecciones de la ciudad de Pereira; teniendo como objetivo general analizar la relevancia que tiene el E-Commerce para el desarrollo comercial del sector confecciones de la ciudad de Pereira. Con el análisis de información de carácter primario y secundario, se podrá establecer si esta novedosa tendencia basada en las TIC, es una estrategia fundamental para que las empresas del mencionado sector mejoren sus resultados comerciales.

Luego del desarrollo de la investigación, se permite exponer que el E-commerce aprueba replantear los objetivos en la empresa con un claro direccionamiento estratégico, facilitando crear nuevos productos y mercados, diferentes canales de distribución, reducir el coste de las actividades empresariales y favorecer la apertura de nuevos mercados (Fernández, 2015). Cabe resaltar que el E-commerce está teniendo un papel importante en las empresas y es fundamental para la globalización, ya que tiene un alcance con los clientes a nivel mundial y no solamente local. La necesidad de llevar un mercado nacional al exterior, convirtió al comercio electrónico en una herramienta favorable para las empresas, obteniendo de esta forma un crecimiento para la comercialización; llevando a la constitución de entidades para promover, regular y controlar el comercio electrónico. En Colombia se constituyó la entidad CCCE Cámara Colombiana de Comercio Electrónico (Mazuera, 2012).

Las empresas que lo implementan logran tener ventajas competitivas frente al comercio tradicional; su inversión se basa en la creación de programas, redes y sistemas que permiten dar a conocer un bien y/o producto al mundo. Lo anterior reafirma la intención de la presente investigación, ya que ella proporciona a los empresarios del sector un conocimiento más a fondo sobre los elementos que les puede brindar.

Comercio Electrónico; CLAVE

E-Commerce; Globalización;

Marketing Digital;

Sector Confecciones

1 Juliana M. Arias Molina, Risaralda, Fundación Universitaria del Areandina, Pereira, Colombia. Correo electrónico: jarias86@estudiantes.areandina.edu.co

2 Valeria Parra Flórez, Risaralda, Fundación Universitaria del Areandina, Pereira, Colombia. Correo electrónico: vparra9@estudiantes.areandina.edu.co 


\title{
Relevancia del E-commerce para el sector confecciones de la ciudad de Pereira
}

\begin{abstract}
This document sets out a proposal to try to determine the importance of E-commerce for improving the marketing of products in the confections sector of the city of Pereira; having as a general objective, to analyze the relevance of E-Commerce to the commercial development of the confections sector of the city of Pereira. With the analysis of primary and secondary information, it will be possible to establish whether this novel Tics-based trend is a key strategy for companies in the sector to improve their business performance.
\end{abstract}

After the development of the research it is possible to explain that e-commerce allows to rethink the objectives in the company with a clear strategic direction, facilitating the creation of new products and markets, new distribution channels, reduce the cost of business activities and encourage the opening of new markets (Fernández, 2015). It should be noted that E-commerce is playing an important role in businesses and is fundamental to globalization as it has a reach with customers globally and not only locally. The need to bring a domestic market abroad made e-commerce a business-friendly tool, thus achieving growth for this form of commercialisation; also establishing entities to promote, regulate and control electronic commerce, in Colombia the entity CCCE Colombian Chamber of Electronic Commerce was established (Mazuera, 2012).

The companies that implement it manage to have competitive advantages over traditional trade; its investment is based on the creation of programs, networks and systems that allow to make a good/product known to the world. This reaffirms the intention of this investigation, as it provides entrepreneurs in the sector with a more in-depth knowledge of the elements it can provide them.

Electronic Commerce;

E-commerce; Globalization;

Digital marketing;

Apparel Sector 


\section{INTRODUCCIÓN}

Con la presente investigación se trata de determinar la relación de la red informática de comunicación mundial respecto a las actividades comerciales de las empresas del sector confecciones de la ciudad de Pereira y exponer la viabilidad que tiene el comercio electrónico como estrategia de mejoramiento de los procesos comerciales en dicho sector. En la primera parte de la investigación se tratarán de establecer las funcionalidades y beneficios que puede generar el E-commerce para las empresas del sector confección de la ciudad de Pereira.

En la actualidad se pueden encontrar múltiples mecanismos de venta, la necesidad de desarrollar un artilugio para llegar a los clientes, generando una experiencia útil y cómoda donde los consumidores adquieran sus productos y servicios de forma fácil y rápida, es una de las principales estrategias que deben implementar las empresas a la hora de abordar el cliente. Por esta razón el interés de llevar a cabo el desarrollo de la investigación, radica en la necesidad que tienen las empresas de ser competitivas y de llegar al cliente de una manera vehemente y eficiente.

La transformación digital ya no es una opción para las empresas, es una obligación, como lo afirma Juan Carlos Mejía Llano en su ponencia sobre "transformación digital: incorpore el mundo digital al ADN de su empresa". Hoy en día es fundamental la implementación del desarrollo digital del marketing y las diferentes estrategias de distribución que nos ofrece el internet para llegar al consumidor final, ya que las empresas del sector confecciones se desarrollan en un plano B2C.

\section{MÉTODO}

La investigación es de tipo cuantitativo con un alcance descriptivo transversal. Se desarrollará mediante el análisis de información de carácter secundario obtenida a través de la revisión bibliográfica y de antecedentes; así mismo, se utilizará información de carácter primario adquirida de los empresarios del sector, mediante la aplicación de un instrumento de recolección de información tipo encuesta.

\section{DISCUSIÓN}

Algunos textos como "El comercio electrónico una guía completa para gestionar las ventas online" Escrito por Ignacio Somalo, afirma que: "Con el comercio electrónico e implementando las nuevas tecnologías se puede tener no solo un reconocimiento nacional sino también global". Por otra parte, los beneficios de las empresas se aprecian en la reducción e incluso eliminación de los intermediarios de la cadena de distribución, además de permitir más cercanía con el cliente, implementando mayor confianza del consumidor. 
Entre las estrategias que se implementan en el marketing digital, se puede reflejar principalmente el énfasis que se tiene hacia generar una relación más amena, desarrollando mayor confianza por parte del consumidor.

El marketing digital tiene múltiples funcionalidades puesto que reduce costos, tanto para el consumidor como para el vendedor. Gracias al avance de las nuevas tecnologías, las empresas pueden aprovechar las ventajas competitivas que genera esta herramienta. Por otra parte, cabe resaltar la importancia del uso del internet y los medios de comunicación en la gestión comercial, ya que en la actualidad se implementa de manera sustancial y viable el tema de los influenciadores, que ayudan al reconocimiento y difusión del producto. Herramientas como Instagram, Facebook entre otras, hoy en día son herramientas fundamentales para empresas que desarrollan el comercio electrónico (Izard). Lo anterior reafirma la intención de la presente investigación, ya que ella proporciona a los empresarios del sector, un conocimiento más a fondo sobre los elementos que les puede brindar.

Los comerciantes que pretenden integrarse a este nuevo ámbito y lo desconocen, pueden observar la gran relevancia que tiene el someter a su negocio en esta nueva adaptación de comercio, ya que las empresas que no se adaptan a las necesidades del mercado, caen en el error de no maximizar sus ventas, sino que, por el contrario, con el paso del tiempo tienden a bajar.

Es novedoso, por ser la actual tendencia que se está generando debido a la gran población de la generación Millennials y los Zetas, que son una comunidad con un nuevo enfoque en la tecnología y las compras Online de los productos necesarios de cualquier parte del mundo. También es innovador que las empresas y negociadores ya no precisen tener un espacio físico, sino un celular y redes sociales para poder vender sus productos. Quien no haga uso de esta nueva técnica de venta, quizás no llegue a tener el mismo impacto con clientes no solamente locales; sino nacionales e internacionales.

\section{CONCLUSIONES}

Tras el desarrollo de la investigación, se espera obtener información importante que permita a las empresas del sector confecciones de la ciudad de Pereira la toma de decisiones, en cuanto a la implementación de estrategias de E-commerce para la infraestructura, tiempos de distribución, costos y comercialización de sus productos.

Esta investigación podrá ofrecer herramientas para los empresarios del sector confecciones de la ciudad de Pereira, para que puedan mejorar la situación de la comercialización de sus empresas, proporcionándoles al usuario comodidad, confiabilidad, satisfacción y reducción de costos. Además, la información suministrada en la investigación servirá de ayuda para los empresarios que no han implementado el E-commerce en sus negocios y que están desaprovechando la reciente oportunidad que se ha venido posicionado entre los consumidores. 
En el terreno ambiental, se le puede brindar un mecanismo a los empresarios para que puedan implementar el comercio electrónico como un factor que beneficie el medio ambiente, en el cual el E-commerce puede ser un potente aliado de la naturaleza, ya que, gracias a la implementación del internet, se promulga un ahorro importante en cuanto a materias primas y energía como afirma Raúl Morales.

1. Castro R. Estefanía; González R. Martha L.; López N. Rebeca. (2013); Comercio electrónico: en la era de la globalización. Esic Editorial, Ideas Propias Editorial, Internacional Thomson Editores, Editor Dikinson.

2. Torre Gustavo S.; Corned Darío G. (2013); Fundamentos del comercio electrónico. Universidad virtual de Quilmes. Argentina.

3. González M. Ricardo. (2012); Aprovechamiento del e-commerce, como medio para que las pymes nuevas o ya constituidas, puedan expandir su negocio. Pontificio Universidad Javeriana. Colombia

4. Toro N. Edna B. (2009); Crecimiento del comercio electrónico en Colombia en los años 2002 a 2009. UNAD Florida. Colombia.

5. Portillo Antonio F. (2015); Comercio electrónico (e-commerce) "aproximación bibliométrica y económica”. Universidad de Extremadura, Escuela Politécnica. España.

6. Anteportamlatinam V. Jose M. (2014); Relevancia del E-commerce para la empresa actual. Universidad de Valladolid. Soria, España.

7. Garcilla P. Rafael; Gil R. Juan; Merino Juan A; Somalo I. (2011); El libro del comercio electrónico.Esic Editorial .España.

8. Seoane B. Eloy. (2005); La nueva era del comercio: el comercio electrónico. Ideas propias editorial. España.

9. Ramos J. (2017); Ecommerce 2.0.

10. Castaño Juan J.; Jurado S. (2016); Comercio electrónico. Editex. España.

11. Silva M. Roxana. (2009); Beneficios del comercio electrónico. Universidad Católica Boliviana San Pablo. Bolivia.

12. Laudon C. Kenneth; Guercio T. Carol. (2016); E-Commerce. Business, technology, society. Pearson. Estados Unidos. 
13. Frasquet D. Marta; Mollá D. Alejandro; Ruiz M. María E. (2012); Factores determinantes y consecuencias de la adopción del comercio electrónico B2C: una comparativa internacional. España.

14. I. A. Rodríguez del Bosque Á; Herrero Crespo. (2008); Antecedentes de la utilidad percibida en la adopción del comercio electrónico entre particulares y empresas. España.

15. Martínez M. Dolores; Soledad M. (2008); Modelos para la gestión de precios de un negocio en la red. Asociación Española de profesores universitarios de Matemática aplicada a la economía y la empresa (ASEPUMA). España.

16. Pereira S. (2011); E-Commerce and business caase of a US E-Commerce firm. Universidad politécnica de Catalunya. Estados Unidos.

17. Cabal C. Claudia C. (2013); Análisis y modelización de la adaptación de los sistemas de recomendación en el comercio electrónico. Universidad internacional de Andalucía. España.

18. Albornoz María M. (2012); La falta de confianza en el comercio electrónico. Centro de investigación y docencia económicas, División de estudios Jurídicos. México.

19. Fernández P. Antonio; Cruz S. María; Hernández M. Ricardo; Jiménez Héctor V. (2015); La importancia de la innovación en el comercio electrónico. Universidad Bussines review.

20. Miralbell I. Oriol. (2014); Redes sociales, economía y empresa. Universitat Oberta Catalunya (Oikonomics, revista de los estudios de economía y empresa). Cataluña. 